MATHEMATICS OF COMPUTATION

Volume 76, Number 257, January 2007, Pages 323-337

S 0025-5718(06)01882-5

Article electronically published on October 11, 2006

\title{
ON THE DISTRIBUTION OF ZEROS OF THE HURWITZ ZETA-FUNCTION
}

\author{
RAMŪNAS GARUNKŠTIS AND JÖRN STEUDING
}

\begin{abstract}
Assuming the Riemann hypothesis, we prove asymptotics for the sum of values of the Hurwitz zeta-function $\zeta(s, \alpha)$ taken at the nontrivial zeros of the Riemann zeta-function $\zeta(s)=\zeta(s, 1)$ when the parameter $\alpha$ either tends to $1 / 2$ and 1 , respectively, or is fixed; the case $\alpha=1 / 2$ is of special interest since $\zeta(s, 1 / 2)=\left(2^{s}-1\right) \zeta(s)$. If $\alpha$ is fixed, we improve an older result of Fujii. Besides, we present several computer plots which reflect the dependence of zeros of $\zeta(s, \alpha)$ on the parameter $\alpha$. Inspired by these plots, we call a zero of $\zeta(s, \alpha)$ stable if its trajectory starts and ends on the critical line as $\alpha$ varies from 1 to $1 / 2$, and we conjecture an asymptotic formula for these zeros.
\end{abstract}

\section{Motivation}

Let, as usual, $s=\sigma+i t$ denote a complex variable and define $\mathrm{e}(z)=\exp (2 \pi i z)$. For $\sigma>1$, the Hurwitz zeta-function is given by

$$
\zeta(s, \alpha)=\sum_{n=0}^{\infty} \frac{1}{(n+\alpha)^{s}},
$$

where $\alpha$ is a parameter from the interval $(0,1]$. The Hurwitz zeta-function can be continued analytically to the whole complex plane except for a simple pole at $s=1$ with residue 1 . For $\alpha=1$ the Hurwitz zeta-function becomes the Riemann zeta-function $\zeta(s):=\zeta(s, 1)$ which is of great importance in number theory. The as-yet unsolved Riemann hypothesis $(\mathrm{RH})$ states that all nontrivial (nonreal) zeros of $\zeta(s)$ lie on the critical line $\sigma=1 / 2$, or equivalently, that the $\zeta(s)$ does not vanish in the half-plane $\sigma>1 / 2$.

As a matter of fact, we have further that

$$
\zeta(s, 1 / 2)=\left(2^{s}-1\right) \zeta(s) .
$$

The second author showed that besides $\alpha=1 / 2,1$ there are no identities of this type; more precisely, in [10] it was proved that $\zeta(s, \alpha) / \zeta(s)$ is entire if and only if $\alpha=1 / 2$ or 1 .

The distribution of zeros of $\zeta(s, \alpha)$ as a function of $s$ depends drastically on the parameter $\alpha$. For instance, the Hurwitz zeta-function given by (1) vanishes for $s=2 \pi i k / \log 2, k \in \mathbb{Z}$, and all other nonreal zeros are expected to lie on the critical line $\sigma=1 / 2$ (by RH). However, this example is somehow special.

Received by the editor March 3, 2005 and, in revised form, October 4, 2005.

2000 Mathematics Subject Classification. Primary 11M35, 11M26.

The first author is partially supported by a grant from the Lithuanian State Science and Studies Foundation and also by INTAS grant no. 03-51-5070. 
It is known that for any $1 / 2<\sigma_{1}<\sigma_{2}<1$ and any transcendental or rational $\alpha \neq 1 / 2,1$ the function $\zeta(s, \alpha)$ has more than $c T$ zeros in the rectangle $\sigma_{1} \leq \sigma \leq \sigma_{2}$, $|t| \leq T$, where $c$ is a positive constant depending on $\sigma_{1}, \sigma_{2}$ and $\alpha$ (see Karatsuba and Voronin [9] or Gonek 8]). This is also expected to hold for algebraic irrational $\alpha$ (see [5] and Corollary 3 of [7]). Thus the analogue of $\mathrm{RH}$ for $\zeta(s, \alpha)$ fails for generic $\alpha \neq 1 / 2,1$. In this note we are concerned with the change in the distribution of zeros of $\zeta(s, \alpha)$ while $\alpha$ tends to $1 / 2$ and 1 , respectively.

By partial summation,

$$
\zeta(s, \alpha)=\frac{1}{\alpha^{s}}+\frac{1}{(1+\alpha)^{s}}+\frac{1}{s-1}\left(\frac{3}{2}+\alpha\right)^{1-s}+s \int_{3 / 2}^{\infty} \frac{1 / 2-\{u\}}{(u+\alpha)^{s+1}} \mathrm{~d} u,
$$

valid for $\sigma>0$, where $\{u\}$ denotes the fractional part of a real number $u$ (see Karatsuba and Voronin [9]). It is easy to see that the last integral converges uniformly for $s$ from any compact subset of the half-plane $\sigma>0$ and arbitrary $\alpha$. It thus follows that $\zeta(s, \alpha)$ is a continuous function in $s \neq 1$ and $\alpha$. Now assume RH. Then, by (1), for any $T$ and any $\delta>0$, there exits a positive constant $c=c(T, \delta)$ such that all nontrivial zeros $\varrho_{\alpha}=\beta_{\alpha}+i \gamma_{\alpha}$ of all Hurwitz zeta-functions $\zeta(s, \alpha)$ with $|1 / 2-\alpha| \leq c$, which have imaginary part $\left|\gamma_{\alpha}\right| \leq T$, satisfy either $\left|\beta_{\alpha}-1 / 2\right| \leq \delta$ or $\left|\beta_{\alpha}-0\right| \leq \delta$. (This phenomenon is illustrated by Figure 3 in Section 3.) It is natural to ask how small $c$ must be such that the zeros $\varrho_{\alpha}$ with $\left|\gamma_{\alpha}\right| \leq T$ cluster around the lines $\sigma=1 / 2,1$. It seems rather difficult to study the continuity of the zeros of $\zeta(s, \alpha)$ with respect to the parameter directly. Instead we consider the sum of values $\zeta(s, \alpha)$ taken at the nontrivial zeros of the Riemann zeta function when the parameter $\alpha$ tends to 1 and $1 / 2$, respectively. The obtained results are presented in the next section. The third section is devoted to empirical calculations of the Hurwitz zeta-function zeros.

\section{Statement of Results}

In 2 Fujii started to consider sums of values of Hurwitz zeta-functions taken at the nontrivial zeros $\varrho$ of $\zeta(s)$. Using an approximate functional equation, he obtained under assumption of $\mathrm{RH}$ that, for fixed $\alpha \neq 1$,

$$
\sum_{0<\gamma \leq T} \zeta(\varrho, \alpha)=-\left(\Lambda\left(\frac{1}{\alpha}\right)+L(1,-\alpha)\right) \frac{T}{2 \pi}+O\left(T^{\frac{9}{10}} \log T\right),
$$

where

$$
\Lambda(x):=\left\{\begin{array}{cl}
\log p & \text { if } x=p^{k}, \text { where } p \in \mathbb{P}, k \in \mathbb{N}, \\
0 & \text { otherwise, }
\end{array}\right.
$$

is the von Mangoldt $\Lambda$-function, $\mathbb{P}$ denotes the set of prime numbers, and

$$
L(s, \alpha):=\sum_{n=1}^{\infty} \frac{\mathrm{e}(\alpha n)}{n^{s}}
$$

note that this series is convergent for $\sigma>0$ if $\alpha$ is not an integer. In view of (11) the main term in (2) vanishes if $\alpha=1 / 2$; in fact, it is also not difficult to prove the converse which yields the claim on the entireness of $\zeta(s, \alpha) / \zeta(s)$ from the previous section. In [10] the second author showed that Fujii's formula (2) holds unconditionally with the error term $O\left(T^{1-c(\log T)^{-2 / 3}}\right)$, where $c$ is an absolute positive constant. Our first aim is to improve Fujii's error term under assumption of $\mathrm{RH}$. 
Theorem 1. Assuming RH, formula (2) is valid with the error term $O\left(T^{\frac{1}{2}+\frac{16}{237}+\varepsilon}\right)$ (note that $\frac{1}{2}+\frac{16}{237}=0.568 \ldots$ ). Assuming GRH (i.e., the analogue of $R H$ for Dirichlet $L$-functions), this error term can be replaced by $O\left(T^{\frac{1}{2}+\varepsilon}\right)$ if $\alpha$ is rational.

Our main interest is the behaviour of Fujii's sum as the parameter $\alpha$ tends either to $1 / 2$ or to 1 .

Theorem 2. Assume RH. For $2 \alpha-1=o(1 / T)$, as $T \rightarrow \infty$,

$$
\begin{aligned}
\sum_{0<\gamma \leq T} \zeta(\varrho, \alpha)= & \frac{\log 2}{2 \pi i}\left(\frac{1}{2 \alpha}+O\left(\frac{|2 \alpha-1|}{\log T}\right)\right)\left(\frac{1}{2 \alpha-1}-\frac{1}{2}+O(|2 \alpha-1|)\right) \\
& \times\left(\exp (-i T(2 \alpha-1))-1+O\left(\frac{1}{T}\right)\right) \\
& +\frac{T}{4 \pi}(\log 2+\log (1-\cos 2 \pi \alpha)-i \pi(2 \alpha-1))+O\left(T^{\frac{1}{2}+\frac{16}{237}+\varepsilon}\right) .
\end{aligned}
$$

In particular,

$$
\begin{aligned}
\sum_{0<\gamma \leq T} \zeta(\varrho, \alpha)= & i \frac{\log 2}{4 \pi}(2 \alpha-1) T^{2}+\frac{\log 2}{12 \pi}(2 \alpha-1)^{2} T^{3} \\
& +O\left(|2 \alpha-1|^{3} T^{4}+T^{\frac{1}{2}+\frac{16}{237}+\varepsilon}\right) .
\end{aligned}
$$

The situation is slightly different if $\alpha$ tends to 1 . Similar to Theorem 2 one might expect Fujii's sum to be small. However, the series $L(s,-1)$ in (2) coincides with $\zeta(s)$, so it has a simple pole at $s=1$ and the sum should be big. The following theorems reflect these observations.

Theorem 3. Assume RH. Let $(1-\alpha)^{-1}=T(\log T)^{\beta}$, with $\beta \geq 0$. Then, as $T \rightarrow \infty$,

$$
\begin{aligned}
\sum_{0<\gamma \leq T} \zeta(\varrho, \alpha)= & -\frac{i}{4 \pi} T(\log T)^{1-\beta}-\frac{1}{12 \pi} T(\log T)^{1-2 \beta} \\
& +O\left(T+T(\log T)^{1-3 \beta}\right) .
\end{aligned}
$$

Theorem 4. Assume RH. Let $0<\delta \leq \alpha \leq 1$. Then, as $T \rightarrow \infty$,

$$
\sum_{0<\gamma \leq T} \zeta(\varrho, \alpha) \ll_{\delta} \min \{1-\alpha,|2 \alpha-1|\} T^{2} \log T,
$$

uniformly in $\alpha$.

The fourth section contains the proof of the first three theorems, and the fifth section gives the proof of Theorem 4 .

\section{Illustrations}

Figure 1 shows the zeros and the absolute value of the Hurwitz zeta-function $\zeta(s, \alpha)$ for $\alpha$ near to 1 and $1 / 2$, respectively. This particular example suggests that the same perturbation (namely 0.0008 ) for $\alpha \approx 1$ and $\alpha \approx 1 / 2$ produce larger perturbations for the zeros of $\zeta(s, \alpha)$ with $\alpha$ near to $1 / 2$. On first sight this seems to be different from what Theorem 2 and 3 predict. Possibly this can be explained by Figure 2, which shows the dependence on $\alpha$ for the sums

$$
\sum_{0<\gamma<1010}|\zeta(\varrho, \alpha)| \quad \text { and } \quad\left|\sum_{0<\gamma<1010} \zeta(\varrho, \alpha)\right|
$$



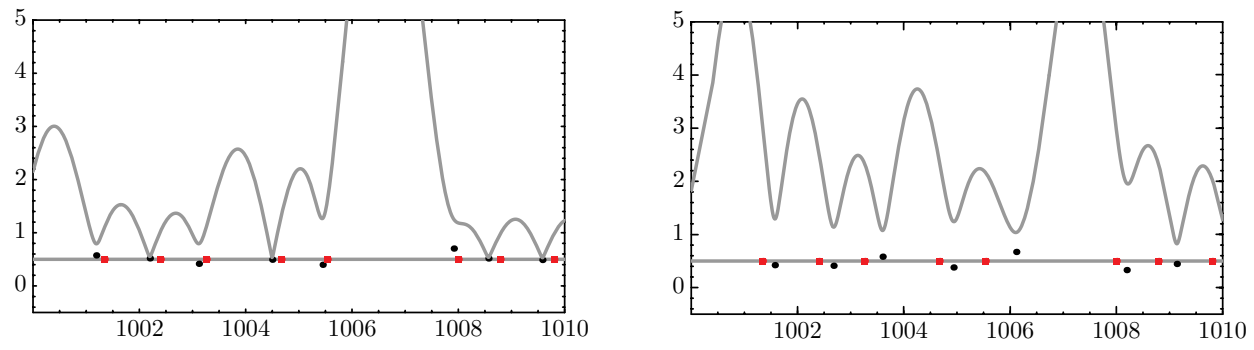

Figure 1. The left graphic corresponds to the case $\alpha=0.9992$, the right to $\alpha=0.5008$. In both pictures the graph is given by $|\zeta(1 / 2+i t, \alpha)|+1 / 2,1000 \leq t \leq 1010$. Squares indicate the zeros of $\zeta(s)$ and dots those of the corresponding $\zeta(s, \alpha)$, lying above or below the straight line $\sigma=1 / 2$ according to having a real part less than or larger than $1 / 2$. In the right graphic we do not draw zeros of $\zeta(s, 0.5008)$ which are clustered around the line $\sigma=0$.
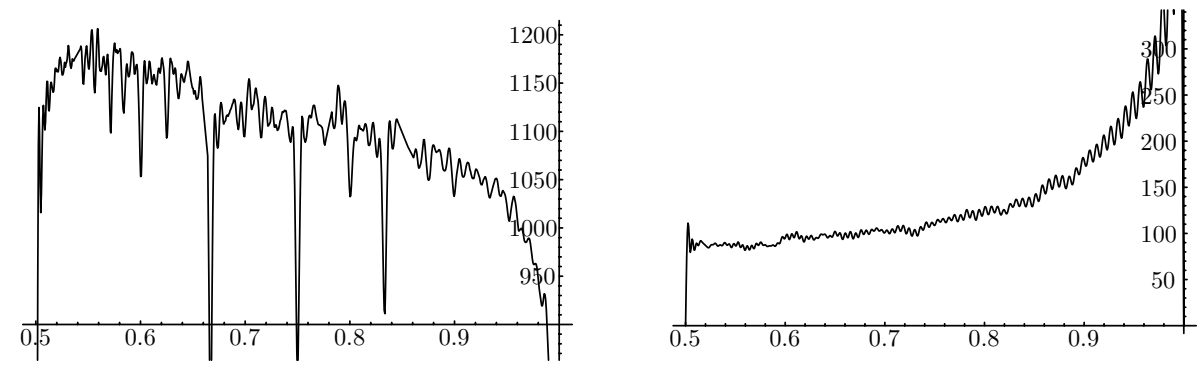

FiguRE 2. The left picture shows the graph of $\sum_{0<\gamma<1010}|\zeta(\varrho, \alpha)|$, the right one the graph of $\left|\sum_{0<\gamma<1010} \zeta(\varrho, \alpha)\right|, 0.5<\alpha<1$.

for $\alpha \in(1 / 2,1)$. The first sum is larger for $\alpha$ near to $1 / 2$ while the second sum is larger for $\alpha$ near 1 . One of the motivations for this paper was Figure 3 , where the trajectories of several zeros of $\zeta(s, \alpha)$ are shown, as $\alpha$ varies in the range $0.5 \leq \alpha \leq 1$. For example, it shows that the trajectories which start at the 30th and the 33rd zeros of $\zeta(s)=\zeta(s, 1)$ end at zeros of $\zeta(s, 1 / 2)$ on the line $\sigma=1$. We believe that all trajectories which start at nontrivial zeros of the Riemann zeta-function end at zeros of $\zeta(s, 1 / 2)$ lying on either $\sigma=1$ or the critical line $\sigma=1 / 2$. We call a zero $\varrho$ of $\zeta(s)$ stable if its trajectory ends on the critical line as $\alpha \rightarrow 1 / 2$; otherwise the zero is called unstable. Denoting the zeros of $\zeta(s)$ with positive ordinate by $\varrho_{n}=\beta_{n}+i \gamma_{n}$ (in ascending order), we find among the first 500 zeros the following unstable zeros, indicated by their index $n$ :

$1,3,6,9,13,17,21,26,30,33,40,44,50,54,61,67,70,78,79$, $90,93,101,109,112,117,124,134,139,147,149,153,165,167$, $175,186,189,197,201,214,218,219,234,235,240,253,255,266$, $270,275,282,288,300,313,317,334,342,344,355,359,361,371$, $384,387,394,409,418,422,434,444,458,476,488,492,493,499$, 500 . 


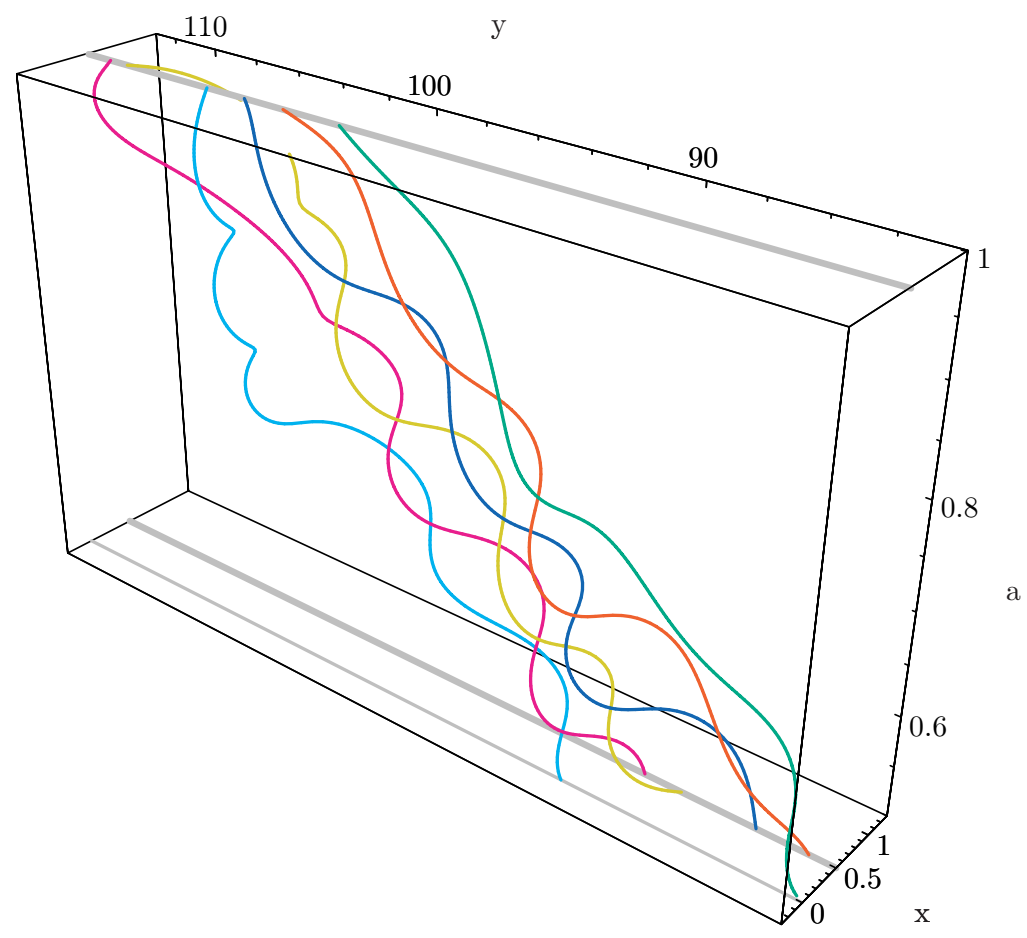

FiguRE 3. Trajectories of several zeros of $\zeta(s, \alpha), 0.5 \leq \alpha \leq 1$; starting with the 30th zero of $\zeta(s)=\zeta(s, 1)$ and ending with the 35 th.

Figure 3 suggest that the zeros for $\alpha=1$ should migrate to zeros of a smaller imaginary part at $\alpha=1 / 2$. The number of nontrivial zeros of $\zeta(s)$ and $\zeta(s, 1 / 2)$ up to $T$ is asymptotically equal to

$$
\frac{T}{2 \pi} \log \frac{T}{2 \pi e}+O(\log T) \quad \text { and } \quad \frac{T}{2 \pi} \log \frac{T}{2 \pi e}+\frac{T}{2 \pi} \log 2+O(\log T),
$$

respectively (see [6]), and $\frac{T}{2 \pi} \log 2+O(1)$ many of these zeros of $\zeta(s, 1 / 2)$ lie on the line $\sigma=0$. Therefore, we may expect that the number of unstable zeros up to $T$ is asymptotically equal to

$$
T \frac{\log 2}{2 \pi}\left(1-\frac{\log 2}{\log \frac{T}{2 \pi e}}\right) ;
$$

on average, we conjecture that about

$$
\frac{1}{\log 2} \log \frac{T}{2 \pi e}
$$

stable zeros lie in between two consecutive unstable zeros with an imaginary part approximately equal to $T$. We do not expect that there are many pairs of consecutive unstable zeros. Among the first 500 zeros (with positive real part) we found only five pairs of such unstable twins:

78,$79 ; 218,219 ; 234,235 ; 492,493$ and $499,500$. 
The computations in this section are based on numerical solutions of the differential equation

$$
\frac{\partial z_{0}(\alpha)}{\partial \alpha}=-\frac{\frac{\partial \zeta(z, \alpha)}{\partial \alpha}}{\frac{\partial \zeta(z, \alpha)}{\partial z}},
$$

where $z=z_{0}(\alpha), \zeta\left(z_{0}(\alpha), \alpha\right)=0$. For initial conditions the zeros of $\zeta(s, 1)$ were used.

\section{The method of Conrey, Ghosh, and GoneK}

The proofs of the first three theorems rely on the method of Conrey, Ghosh, and Gonek [1, namely the idea to interpret the sum in question as a sum of residues, resp. a contour integral

$$
\sum_{0<\gamma<T} \zeta(s, \alpha)=\frac{1}{2 \pi i} \int_{\mathfrak{C}} \frac{\zeta^{\prime}}{\zeta}(s) \zeta(s, \alpha) \mathrm{d} s,
$$

which can be evaluated by Gonek's lemma. First of all, we have to choose an appropriate path of integration $\mathfrak{C}$ according to the condition of summation. Note that the first nontrivial zero with positive imaginary part is $1 / 2+i 14.13$ to two decimal places. By the Riemann-von Mangoldt formula for the number of nontrivial zeros of $\zeta(s)$,

$$
N(T):=\#\{\varrho=\beta+i \gamma: 0<\gamma \leq T\}=\frac{T}{2 \pi} \log \frac{T}{2 \pi e}+O(\log T),
$$

it follows that the zeros $\varrho$ cannot lie too dense: for any given $T_{0}>1$ there exists a $T \in\left(T_{0}, T_{0}+1\right]$ such that

$$
\min _{\gamma}|T-\gamma| \gg \frac{1}{\log T} .
$$

Now let $a=1+1 / \log T$ and define the contour $\mathfrak{C}$ to be the rectangle with vertices $a+i, a+i T, 1-a+i T, 1-a+i$. By the calculus of residues (3) holds and, say,

$$
\begin{aligned}
& \frac{1}{2 \pi i} \int_{\mathfrak{C}} \frac{\zeta^{\prime}}{\zeta}(s) \zeta(s, \alpha) d s \\
& =\frac{1}{2 \pi i}\left(\int_{a+i}^{a+i T}+\int_{a+i T}^{1-a+i T}+\int_{1-a+i T}^{1-a+i}+\int_{1-a-i}^{a+i}\right) \frac{\zeta^{\prime}}{\zeta}(s) \zeta(s, \alpha) \mathrm{d} s \\
& =: \sum_{j=1}^{4} \mathfrak{I}_{j} .
\end{aligned}
$$

Let $0<\delta<1 / 2$ be fixed. We shall evaluate the integrals $\mathfrak{I}_{j}, j=1, \ldots, 4$, uniformly in $\alpha$ for the range $\delta \leq \alpha \leq 1$. The case of fixed $\alpha$ corresponds to Theorem 1] the case of $\alpha \rightarrow 1 / 2$ to Theorem 2 , and, finally, $\alpha \rightarrow 1$ to Theorem 3 .

In the half-plane of absolute convergence $\sigma>1$ we may rewrite the integrand in (6) as a Dirichlet series and interchange summation and integration. It is easily seen that

$$
\frac{\zeta^{\prime}}{\zeta}(s)=-\sum_{m=2}^{\infty} \frac{\Lambda(m)}{m^{s}}
$$


This leads to

$$
\mathfrak{I}_{1}=-\frac{1}{2 \pi} \sum_{m=2}^{\infty} \sum_{n=0}^{\infty} \frac{\Lambda(m)}{(m(n+\alpha))^{a}} \int_{1}^{T} \frac{\mathrm{d} t}{(m(n+\alpha))^{i t}} .
$$

By the Laurent expansions at $s=1$,

$$
\begin{gathered}
\frac{\zeta^{\prime}}{\zeta}(s)=\frac{-1}{s-1}+\gamma+O(s-1), \\
\zeta(s, \alpha)=\frac{1}{s-1}+\gamma(\alpha)+O(s-1),
\end{gathered}
$$

valid for $s \rightarrow 1$, we get

$$
\begin{aligned}
\sum_{m=2}^{\infty} \sum_{n=0}^{\infty} \frac{\Lambda(m)}{(m(n+\alpha))^{a}} & \ll \sum_{m=2}^{\infty} \sum_{n=0}^{\infty} \frac{\Lambda(m)}{(m(n+\delta))^{a}} \\
& \ll \frac{\zeta^{\prime}}{\zeta}(a) \zeta(a, \delta) \ll(\log T)^{2} .
\end{aligned}
$$

For fixed $\alpha$ the integral in (17) is bounded unless $n=0$ and $m \alpha=1$. Thus

$$
\mathfrak{I}_{1}=-\Lambda\left(\frac{1}{\alpha}\right) \frac{T}{2 \pi}+O\left((\log T)^{2}\right) .
$$

For $\alpha \rightarrow 1 / 2($ but $\neq 1 / 2$ ) we have

$$
\int_{1}^{T} \frac{\mathrm{d} t}{(2 \alpha)^{i t}}=\int_{0}^{T} \frac{\mathrm{d} t}{(2 \alpha)^{i t}}+O(1)=\frac{1-\exp (-i T \log (2 \alpha))}{i \log (2 \alpha)}+O(1)
$$

and

$$
\mathfrak{I}_{1}=\frac{\exp (-i T \log (2 \alpha))-1}{2 \pi i(2 \alpha)^{a} \log (2 \alpha)} \log 2+O\left((\log T)^{2}\right)
$$

By

$$
\begin{aligned}
& (2 \alpha)^{-a}=\frac{1}{2 \alpha}+O\left(-\frac{|2 \alpha-1|}{\log T}\right) \\
& \log (2 \alpha)=\log (1+2 \alpha-1)=2 \alpha-1+O\left((2 \alpha-1)^{2}\right)
\end{aligned}
$$

and

$$
\frac{1}{\log (2 \alpha)}=\frac{1}{2 \alpha-1}-\frac{1}{2}+O(|2 \alpha-1|)
$$

we obtain

$$
\begin{aligned}
\mathfrak{I}_{1}= & \frac{\log 2}{2 \pi i}\left(\frac{1}{2 \alpha}+O\left(\frac{|2 \alpha-1|}{\log T}\right)\right)\left(\frac{1}{2 \alpha-1}-\frac{1}{2}+O(|2 \alpha-1|)\right) \\
& \times\left(\exp (-i T(2 \alpha-1))-1+O\left(\frac{1}{T}\right)\right)+O\left((\log T)^{2}\right) .
\end{aligned}
$$

Expanding in powers of $T$ and $2 \alpha-1$, this expression is equal to

$$
\begin{aligned}
-\log 2\left(\frac{1}{2 \pi} T-\frac{i}{4 \pi} T^{2}(2 \alpha-1)-\frac{1}{12 \pi} T^{3}(2 \alpha-1)^{2}\right. & \left.+\frac{i}{48 \pi} T^{4}(2 \alpha-1)^{3}\right) \\
& +O\left(T^{5}(2 \alpha-1)^{4}+(\log T)^{2}\right) .
\end{aligned}
$$

Clearly, for $\alpha \rightarrow 1$,

$$
\mathfrak{I}_{1} \ll(\log T)^{2} .
$$


Now we show that the integrand is small on the horizontal paths. For the logarithmic derivative we have the partial fraction decomposition

$$
\frac{\zeta^{\prime}}{\zeta}(s)=\sum_{|t-\gamma| \leq 1} \frac{1}{s-\varrho}+O(\log |t+2|) \quad \text { for } \quad-1 \leq \sigma \leq 2,|t| \geq 1
$$

(for a proof see [9]). With regard to (4) and (5) it follows that

$$
\frac{\zeta^{\prime}}{\zeta}(\sigma+i T) \ll(\log T)^{2} \quad \text { for } \quad-1 \leq \sigma \leq 2, T \geq 1 .
$$

Recall that the Hurwitz zeta-function satisfies the identity

$$
\zeta(1-s, \alpha)=\frac{\Gamma(s)}{(2 \pi)^{s}}\left(\mathrm{e}\left(\frac{s}{4}\right) L(s,-\alpha)+\mathrm{e}\left(-\frac{s}{4}\right) L(s, \alpha)\right) .
$$

Thus, an application of the Phragmen-Lindelöf principle yields the estimate

$$
\zeta(s, \alpha) \ll|t|^{\frac{1}{2}} \log |t+2| \quad \text { for } \quad-\frac{1}{\log T} \leq \sigma \leq 1+\frac{1}{\log T},|t| \geq 1,
$$

uniformly in $\delta \leq \alpha \leq 1$ and $|t| \ll T$. Hence,

$$
\mathfrak{I}_{2}, \mathfrak{I}_{4} \ll T^{\frac{1}{2}}(\log T)^{3} .
$$

It remains to evaluate $\mathfrak{I}_{3}$. Substituting $s \mapsto 1-\bar{s}$, we find that

$$
\overline{\mathfrak{I}}_{3}=-\frac{1}{2 \pi i} \int_{a+i}^{a+i T} \frac{\zeta^{\prime}}{\zeta}(1-s) \zeta(1-s, \alpha) \mathrm{d} s .
$$

Now we shall use functional equations to transform the latter integral into a more suitable expression. Functional equations for $\zeta(s)$ can be written in the form

$$
\zeta(s)=\Delta(s) \zeta(1-s)
$$

where

$$
\Delta(s):=\frac{(2 \pi)^{s}}{2 \Gamma(s) \cos \frac{\pi s}{2}} .
$$

By this and (12) we obtain, say,

$$
\begin{aligned}
\overline{\mathfrak{I}_{3}}= & -\frac{1}{2 \pi i} \int_{a+i}^{a+i T} \frac{\Delta^{\prime}}{\Delta}(s) \frac{\Gamma(s)}{(2 \pi)^{s}} \mathrm{e}\left(-\frac{s}{4}\right) L(s, \alpha) \mathrm{d} s \\
& -\frac{1}{2 \pi i} \int_{a+i}^{a+i T} \frac{\Delta^{\prime}}{\Delta}(s) \frac{\Gamma(s)}{(2 \pi)^{s}} \mathrm{e}\left(\frac{s}{4}\right) L(s,-\alpha) \mathrm{d} s \\
& +\frac{1}{2 \pi i} \int_{a+i}^{a+i T} \frac{\zeta^{\prime}}{\zeta}(s) \frac{\Gamma(s)}{(2 \pi)^{s}} \mathrm{e}\left(-\frac{s}{4}\right) L(s, \alpha) \mathrm{d} s \\
& +\frac{1}{2 \pi i} \int_{a+i}^{a+i T} \frac{\zeta^{\prime}}{\zeta}(s) \frac{\Gamma(s)}{(2 \pi)^{s}} \mathrm{e}\left(\frac{s}{4}\right) L(s,-\alpha) \mathrm{d} s \\
= & \sum_{j=1}^{4} \mathfrak{F}_{j} .
\end{aligned}
$$

We have $\Delta(s) \Delta(1-s)=1$. If $\delta= \pm 1$, then

$$
\frac{\mathrm{e}\left(\delta \frac{s}{4}\right)}{2 \cos \frac{\pi s}{2}}= \begin{cases}O(\exp (-\pi|t|)) & \text { if } \delta t \geq 0 \\ 1+O(\exp (-\pi|t|)) & \text { otherwise. }\end{cases}
$$


Moreover, for $|t| \geq 1$,

$$
\frac{\Delta^{\prime}}{\Delta}(s)=-\log \frac{|t|}{2 \pi}+O\left(\frac{1}{|t|}\right) .
$$

From these estimates it follows that

$$
\mathfrak{F}_{1}=-\frac{1}{2 \pi i} \int_{a+i}^{a+i T} \frac{\Delta^{\prime}}{\Delta}(s) \Delta(1-s) L(s, \alpha) \mathrm{d} s+O(1) .
$$

Furthermore, we find that

$$
\begin{aligned}
& \frac{1}{2 \pi i} \int_{a+i}^{a+i T} \frac{\Delta^{\prime}}{\Delta}(s) \Delta(1-s) L(s, \alpha) \mathrm{d} s \\
= & \int_{1}^{T}\left(-\log \frac{\tau}{2 \pi}+O\left(\frac{1}{\tau}\right)\right) \mathrm{d} \frac{1}{2 \pi i} \int_{a+i}^{a+i \tau} \Delta(1-s) L(s, \alpha) \mathrm{d} s .
\end{aligned}
$$

To evaluate these integrals we shall use a variant of Gonek's lemma.

Lemma 5. Assume that

$$
\sum_{m \leq x}\left|a_{m}\right| \ll x \quad \text { and } \quad b_{n} \ll 1 .
$$

Let $1<c \leq 1+1 / \log \tau$ and $0<\delta<1$, then

$$
\begin{aligned}
& \frac{1}{2 \pi i} \int_{c+i}^{c+i \tau} \Delta(1-s) \sum_{m=1}^{\infty} \frac{a_{m}}{m^{s}} \sum_{n=0}^{\infty} \frac{b_{n}}{(n+\alpha)^{s}} d s \\
& =\sum_{\substack{m \geq 1, n \geq 0 \\
m(n+\alpha) \leq \frac{\tau}{2 \pi}}} a_{m} b_{n} \mathrm{e}(-m(n+\alpha))+O\left(\tau^{\frac{1}{2}}(c-1)^{-2}\right)
\end{aligned}
$$

uniformly in $\alpha \in[\delta, 1]$.

Proof. In [1] it was shown that, for any $r>0$ and any $c_{0} \in(0,2)$,

$$
\frac{1}{2 \pi i} \int_{c+i}^{c+i \tau} \Delta(1-s) r^{-s} \mathrm{~d} s= \begin{cases}\mathrm{e}(-r)+E(r, c) r^{-c} & \text { if } r \leq \frac{\tau}{2 \pi} \\ E(r, c) r^{-c} & \text { otherwise }\end{cases}
$$

uniformly for $c \in\left[c_{0}, 2\right]$, where

$$
E(r, c) \ll \tau^{c-\frac{1}{2}}+\frac{\tau^{c+\frac{1}{2}}}{|\tau-2 \pi r|+\tau^{\frac{1}{2}}} .
$$

Applying this result, we obtain that the integral of the theorem is equal to

$$
\begin{aligned}
& \sum_{\substack{m \geq 1, n \geq 0 \\
m(n+\alpha) \leq \frac{\tau}{2 \pi}}} a_{m} b_{n} \mathrm{e}(-m(n+\alpha)) \\
+ & O\left(\sum_{m \geq 1, n \geq 0}\left(\frac{a_{m} b_{n} \tau^{c-\frac{1}{2}}}{(m(n+\alpha))^{c}}+\frac{a_{m} b_{n} \tau^{c+\frac{1}{2}}}{|\tau-2 \pi m(n+\alpha)|+\tau^{\frac{1}{2}}} \frac{1}{(m(n+\alpha))^{c}}\right)\right) .
\end{aligned}
$$


In order to evaluate the error term we divide the range of summation $m \geq 1, n \geq 0$ into the following three sets:

$$
\begin{array}{ll}
A: & |\tau-2 \pi m(n+\alpha)|>\frac{1}{2} \tau \\
B: & \tau^{\frac{1}{2}} \leq|\tau-2 \pi m(n+\alpha)| \leq \frac{1}{2} \tau \\
C: & |\tau-2 \pi m(n+\alpha)|<\tau^{\frac{1}{2}} .
\end{array}
$$

We find that

$$
\begin{aligned}
\sum_{A} \ll & \tau^{\frac{1}{2}} \sum_{m \geq 1, n \geq 0} \frac{a_{m}}{(m(n+\alpha))^{c}} \ll \tau^{\frac{1}{2}}(c-1)^{-2}, \\
\sum_{B} \ll & \tau^{\frac{1}{2}} \sum_{m \geq 1, n \geq 1} \frac{a_{m}}{(m n)^{c}} \\
& +\tau^{c+\frac{1}{2}} \sum_{m \geq 1} \frac{a_{m}}{m^{c}} \sum_{\tau^{\frac{1}{2}} \leq|\tau-2 \pi m(n+\alpha)| \leq \frac{1}{2} \tau} \frac{1}{|\tau-2 \pi m(n+\alpha)|(m(n+\alpha))^{c}} \\
\ll & \tau^{\frac{1}{2}}(c-1)^{-2}+\tau^{\frac{1}{2}} \sum_{m \geq 1} \frac{a_{m}}{m^{c}} \ll \tau^{\frac{1}{2}}(c-1)^{-2},
\end{aligned}
$$

and

$$
\begin{aligned}
\sum_{C} \ll & \tau^{\frac{1}{2}} \sum_{m \geq 1, n \geq 1} \frac{a_{m}}{(m n)^{c}} \\
& +\tau^{c} \sum_{m \geq 1} \frac{a_{m}}{m^{c}} \sum_{|\tau-2 \pi m(n+\alpha)|<\tau^{\frac{1}{2}}} \frac{1}{(m(n+\alpha))^{c}} \\
\ll & \tau^{\frac{1}{2}}(c-1)^{-2}+\tau^{\frac{1}{2}}(c-1) \sum_{m \geq 1} \frac{a_{m}}{m^{c}} \ll \tau^{\frac{1}{2}}(c-1)^{-2}
\end{aligned}
$$

This proves the lemma.

We continue with the proofs of the theorems. Using Lemma 5 we find for the integral in formula (16) that

$$
\frac{1}{2 \pi i} \int_{a+i}^{a+i \tau} \Delta(1-s) L(s,-\alpha) \mathrm{d} s=\sum_{n \leq \frac{\tau}{2 \pi}} \mathrm{e}(\alpha n)+O\left(\tau^{\frac{1}{2}}(\log T)^{2}\right)
$$

uniformly in $\alpha$ for $\delta \leq \alpha \leq 1$, as $\tau \rightarrow \infty$. The sum on the right-hand side is small for fixed $0<\alpha<1$ and large for $\alpha=1$. Writing $[\alpha]:=\alpha-\{\alpha\}$ for the integral part of $\alpha$, we get

$$
\mathfrak{F}_{1}=\int_{1}^{T}\left(\log \frac{\tau}{2 \pi}+O\left(\frac{1}{\tau}\right)\right) \mathrm{d}\left([\alpha] \frac{\tau}{2 \pi}+O\left(\tau^{\frac{1}{2}}(\log T)^{2}\right)\right) .
$$

Since $[\alpha]$ vanishes exactly for $\alpha \neq 1$, we get for fixed $\alpha$

$$
\mathfrak{F}_{1}=[\alpha] \frac{T}{2 \pi} \log \frac{T}{2 \pi e}+O\left(T^{\frac{1}{2}}(\log T)^{3}\right) .
$$


It is easy to see that, for $\alpha \rightarrow 1 / 2$,

$$
\mathfrak{F}_{1} \ll T^{\frac{1}{2}}(\log T)^{3} .
$$

Next we consider the case $\alpha \rightarrow 1$. First of all, for $|\alpha-1| \ll x^{-1}$,

$$
\begin{aligned}
\sum_{n \leq x} \mathrm{e}(\alpha n)= & \sum_{n \leq x} \mathrm{e}(n(\alpha-1)) \\
= & \sum_{n \leq x}\left(1+2 \pi i n(\alpha-1)-2(\pi n(\alpha-1))^{2}+O\left((n(\alpha-1))^{3}\right)\right) \\
= & {[x]+2 \pi i \frac{1+[x]}{2}[x](\alpha-1)-2(\pi(\alpha-1))^{2} \frac{2[x]^{3}+3[x]^{2}+[x]}{6} } \\
& +O\left([x]^{4}(\alpha-1)^{3}\right) \\
= & x+\pi i x^{2}(\alpha-1)-\frac{2}{3}(\pi(\alpha-1))^{2} x^{3}+O\left(x^{4}(\alpha-1)^{3}+1\right) .
\end{aligned}
$$

Hence

$$
\begin{aligned}
& \frac{1}{2 \pi i} \int_{a+i}^{a+i \tau} \Delta(1-s) L(s,-\alpha) \mathrm{d} s \\
= & \frac{\tau}{2 \pi}+\frac{i}{4 \pi} \tau^{2}(\alpha-1)-\frac{1}{12 \pi} \tau^{3}(\alpha-1)^{2}+O\left(\left((1-\alpha)^{3} \tau^{4}\right)+\tau^{\frac{1}{2}}(\log \tau)^{2}+1\right) .
\end{aligned}
$$

After a short computation this leads to

$$
\begin{aligned}
\mathfrak{F}_{1}= & \frac{T}{2 \pi} \log \frac{T}{2 \pi e}+\frac{i(\alpha-1) T^{2}}{4 \pi} \log \frac{T}{2 \pi e}+\frac{i(\alpha-1) T^{2}}{8 \pi} \\
& -\frac{(\alpha-1)^{2} T^{3}}{12 \pi} \log \frac{T}{2 \pi e}-\frac{(\alpha-1)^{2} T^{3}}{18 \pi} \\
& +O\left((1-\alpha)^{3} T^{4} \log T+T^{\frac{1}{2}}(\log T)^{3}\right) .
\end{aligned}
$$

Putting $(1-\alpha)^{-1}=T(\log T)^{\beta}$, where $\beta \geq 0$, we find that

$$
\begin{aligned}
\mathfrak{F}_{1}= & \frac{T}{2 \pi} \log T+\frac{i}{4 \pi} T(\log T)^{1-\beta}-\frac{1}{12 \pi} T(\log T)^{1-2 \beta} \\
& +O\left(T(\log T)^{1-3 \beta}+T\right) .
\end{aligned}
$$

By the same reasoning we get

$$
\mathfrak{F}_{3}=\frac{1}{2 \pi i} \int_{a+i}^{a+i T} \Delta(1-s) \frac{\zeta^{\prime}}{\zeta}(s) L(s, \alpha) \mathrm{d} s+O(1) .
$$

Lemma 5 yields

$$
\begin{aligned}
& \frac{1}{2 \pi i} \int_{a+i}^{a+i T} \Delta(1-s) \frac{\zeta^{\prime}}{\zeta}(s) L(s, \alpha) \mathrm{d} s \\
= & -\sum_{m n \leq \frac{T}{2 \pi}} \Lambda(m) \mathrm{e}(\alpha n)+O\left(T^{\frac{1}{2}}(\log T)^{2}\right) .
\end{aligned}
$$


Let $U$ be a positive parameter such that $U$ is not the ordinate of any zero of $\zeta(s)$. By Perron's formula,

$$
\begin{aligned}
-\sum_{m n \leq \frac{T}{2 \pi}} \Lambda(m) e(\alpha n)= & \frac{1}{2 \pi i} \int_{a-i U}^{a+i U} \frac{\zeta^{\prime}}{\zeta}(s) L(s, \alpha)\left(\frac{T}{2 \pi}\right)^{s} \frac{\mathrm{d} s}{s} \\
& +O\left(\log T+\frac{T(\log T)^{2}}{U}\right),
\end{aligned}
$$

uniformly in $\alpha$ for $\delta \leq \alpha \leq 1$, as $T \rightarrow \infty$. It follows from the calculus of residues that

$$
\begin{aligned}
& \frac{1}{2 \pi i} \int_{a-i U}^{a+i U} \frac{\zeta^{\prime}}{\zeta}(s) L(s, \alpha)\left(\frac{T}{2 \pi}\right)^{s} \frac{\mathrm{d} s}{s} \\
= & \frac{1}{2 \pi i}\left\{\int_{a-i U}^{b-i U}+\int_{b-i U}^{b+i U}+\int_{b+i U}^{a+i U}\right\} \frac{\zeta^{\prime}}{\zeta}(s) L(s, \alpha)\left(\frac{T}{2 \pi}\right)^{s} \frac{\mathrm{d} s}{s} \\
& +\operatorname{Res}_{s=1} \frac{\zeta^{\prime}}{\zeta}(s) L(s, \alpha)\left(\frac{T}{2 \pi}\right)^{s} \frac{1}{s},
\end{aligned}
$$

where we choose $b=1 / 2+1 / \log T$. The residue is easily seen to be equal to $-L(1, \alpha) T /(2 \pi)$. The integrals contribute to the error term. Here we calculate this error term under RH; the unconditional case is given in [10]. In [4] the first author has proved (unconditionally) that, for any $\varepsilon>0,0<\alpha<1$, and $t \geq 1$,

$$
L\left(\frac{1}{2}+i t, \alpha\right) \ll_{\varepsilon} t^{\frac{32}{205}+\varepsilon}+\frac{1}{\sqrt{\alpha}}+\frac{e^{-\pi t}}{\sqrt{1-\alpha}},
$$

as $t \rightarrow \infty$. From [3] we have, under the same conditions,

$$
L(1+i t, \alpha,) \ll t^{\varepsilon} .
$$

Then, by the Phragmén-Lindelöf principle (see $\S 5.6 .5$ of [11) and $L(s, \alpha)=$ $\overline{L(\bar{s}, 1-\alpha)}$, we derive that for $1 / 2 \leq \sigma \leq 1+\varepsilon, 0<\alpha<1$, and $|t| \geq 1$,

$$
L(s, \alpha) \ll|t|^{\frac{64}{205}(1-\sigma)+\varepsilon}+\frac{1}{\sqrt{\alpha}}+\frac{1}{\sqrt{1-\alpha}} .
$$

It follows from the proof of Theorem 1.1 in Chapter 4 of [6] that, for $0<\alpha<1$ and $|t| \leq 1$,

$$
L\left(\frac{1}{2}+\frac{1}{\log T}+i t, \alpha\right) \ll \frac{1}{\sqrt{\alpha}}+\frac{1}{\sqrt{1-\alpha}} .
$$

Using these bounds together with (11) we get

$$
\int_{a \pm i U}^{b \pm i U} \frac{\zeta^{\prime}}{\zeta}(s) L(s, \alpha)\left(\frac{T}{2 \pi}\right)^{s} \frac{\mathrm{d} s}{s} \ll T U^{-1+\varepsilon}+\frac{T}{U}\left(\frac{1}{\sqrt{\alpha}}+\frac{1}{\sqrt{1-\alpha}}\right)
$$

and

$$
\int_{b-i U}^{b+i U} \frac{\zeta^{\prime}}{\zeta}(s) L(s, \alpha)\left(\frac{T}{2 \pi}\right)^{s} \frac{\mathrm{d} s}{s} \ll T^{\frac{1}{2}} \log ^{2} T\left(U^{\frac{32}{205}+\varepsilon}+\left(\frac{1}{\sqrt{\alpha}}+\frac{1}{\sqrt{1-\alpha}}\right) \log T\right) .
$$


First, let us assume that $\alpha \rightarrow 1 / 2$ or that $\alpha$ is fixed. Choosing $U=T^{\frac{205}{474}}$ we find that, for $\delta \leq \alpha<1-\delta$,

$$
\begin{aligned}
\overline{\mathfrak{F}}_{3} & =-\sum_{m n \leq \frac{T}{2 \pi}} \Lambda(m) e((1-\alpha) n)+O\left(T^{\frac{1}{2}}(\log T)^{2}\right) \\
& =-L(1,1-\alpha) \frac{T}{2 \pi}+O\left(T^{\frac{1}{2}+\frac{16}{237}+\varepsilon}\right) .
\end{aligned}
$$

In view of

$$
L(1,1-\alpha)=-\frac{1}{2}(\log 2+\log (1-\cos 2 \pi \alpha)+i \pi(1-2 \alpha)),
$$

we get, for $\alpha \rightarrow 1 / 2$,

$$
\overline{\mathfrak{F}}_{3}=\frac{T}{2 \pi} \log 2+i \pi(1-2 \alpha) \frac{T}{4 \pi}+O\left(T^{\frac{1}{2}+\frac{16}{237}}+T(1-2 \alpha)^{2}\right) .
$$

Now suppose that $\alpha \rightarrow 1$. In this case we insert the prime number theorem under assumption of $\mathrm{RH}$,

$$
\sum_{m \leq x} \Lambda(m)=x+O\left(x^{\frac{1}{2}}(\log x)^{2}\right)
$$

in (23). We obtain

$$
\begin{aligned}
\overline{\mathfrak{F}}_{3} & =-\sum_{n \leq \frac{T}{2 \pi}} e((1-\alpha) n) \sum_{m \leq \frac{T}{2 \pi n}} \Lambda(m)+O\left(T^{\frac{1}{2}}(\log T)^{2}\right) \\
& =-\sum_{n \leq \frac{T}{2 \pi}}(1+O(n(1-\alpha)))\left(\frac{T}{2 \pi n}+O\left(\left(\frac{T}{n}\right)^{\frac{1}{2}}\left(\log \frac{T}{n}\right)^{2}\right)\right) \\
& =-\frac{T}{2 \pi} \log T+O\left(T+T^{2}(1-\alpha)\right) .
\end{aligned}
$$

as $T \rightarrow \infty$. Taking $(1-\alpha)^{-1}=T(\log T)^{\beta}, \beta \geq 0$, we obtain

$$
\overline{\mathfrak{F}}_{3}=-\frac{T}{2 \pi} \log T+O(T)
$$

In view of (15) we find for $\alpha \in[\delta, 1]$

$$
\mathfrak{F}_{2}, \mathfrak{F}_{4}=O_{\delta}(1) .
$$

Now the first part of Theorem 1 follows by (3), (6), (8), (13), (14), (17), (27), (23). The second part of Theorem 1 follows in the same way as the first part, only in the formula (20) we choose $U=T^{\frac{1}{2}}$, and instead of (22) we use the bound

$$
L\left(\frac{1}{2}+i t, \alpha\right) \ll t^{\varepsilon},
$$

which is valid under GRH, for $\alpha$ is fixed rational number (for the proof see [4). Theorem 2 follows from (3), (6), (9), (13), (14), (18), (23), (24), (25), and (27). Theorem 3 can be obtained from (3), (6), (10), (13), (14), (19), (26)), and (27). 


\section{Proof of Theorem 4}

If $0<\sigma_{0} \leq \sigma \leq 2$ and $2 \pi \leq|t| \leq \pi x$, then

$$
\zeta(s, \alpha)=\sum_{0 \leq n \leq x} \frac{1}{(n+\alpha)^{s}}+\frac{x^{1-s}}{s-1}+O_{\sigma_{0}}\left(x^{-\sigma}\right),
$$

as $x \rightarrow \infty$ (see [9]). For $t \geq 1$, as $n \rightarrow \infty$,

$$
\left|\left(n+\alpha_{2}\right)^{s}-\left(n+\alpha_{1}\right)^{s}\right| \ll \frac{t\left|\alpha_{1}-\alpha_{2}\right|}{n^{1-\sigma}} .
$$

Thus for $\sigma_{0} \leq \sigma \leq 1$, as $t \rightarrow \infty$,

$$
\left|\zeta\left(s, \alpha_{1}\right)-\zeta\left(s, \alpha_{2}\right)\right| \ll t\left|\alpha_{1}-\alpha_{2}\right| .
$$

Hence, under RH,

$$
\begin{aligned}
\sum_{0<\gamma \leq T} \zeta(\varrho, \alpha) & =\sum_{0<\gamma \leq T}\left(\zeta\left(\frac{1}{2}+i \gamma, \alpha\right)-\zeta\left(\frac{1}{2}+i \gamma, \frac{1}{2}\right)\right) \\
& \ll\left|\alpha-\frac{1}{2}\right| \sum_{0<\gamma \leq T} \gamma \ll\left|\alpha-\frac{1}{2}\right| T^{2} \log T, \\
\sum_{0<\gamma \leq T} \zeta(\varrho, \alpha) & =\sum_{0<\gamma \leq T}\left(\zeta\left(\frac{1}{2}+i \gamma, \alpha\right)-\zeta\left(\frac{1}{2}+i \gamma, 1\right)\right) \\
& \ll|\alpha-1| T^{2} \log T,
\end{aligned}
$$

where we have used the Riemann-von Mangoldt formula for a number of nontrivial zeros. Theorem 4 is proved.

\section{ACKNOWLEDGMENTS}

We are very grateful to the anonymous referee for pointing out several mistakes and making suggestions for improvements of our error terms in a previous version of this paper.

We would like to thank Michael Trot for sending us the MATHEMATICA notebook for M. Trotт, "Zeros of the Generalized Riemann Zeta Function as a Function of a", Background image in graphics gallery in Wolfram [12]. See also the webpage http://documents.wolfram.com/v4/MainBook/G.2.22.html.

\section{REFERENCES}

[1] J.B. Conrey, A. Ghosh, S.M. Gonek, Simple zeros of the Riemann zeta-function, Proc. London Math. Soc. 76 (1998), 497-522. MR1616809(99i:11074)

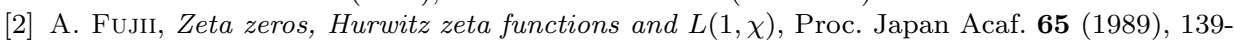
142. MR.1011854 (90i:11088)

[3] R. Garunkštis, Approximation of the Lerch zeta-function, Lith. Math. J. 44(2) (2004), 140-144. MR2116480 (2005j:11060)

[4] R. Garunkštis, Growth of the Lerch zeta-function, Lith. Math. J. 45(1) (2005), 45-46. MR2022961 (2006e:11130)

[5] R. Garunkštis, Note on the zeros of the Hurwitz zeta-function, in: Voronoi's impact on modern science. Book 3: proceedings of the third Voronoi Conference on Number Theory and Spatial Tessellations. Mathematics and its Applications 55 (2005), 10-12.

[6] R. Garunkštis, A. LAURINČIKAS, The Lerch zeta-function, Kluwer, Dordrecht 2002. MR:1979048 (2004c:11161)

[7] R. Garunkštis, J. Steuding, On the zero distributions of Lerch zeta-functions, Analysis 22 (2002), 1-12. MR:1899910(2003a:11115) 
[8] S.M. GoneK, Analytic properties of zeta and L-functions, Ph. D. Thesis, University of Michigan 1979.

[9] A.A. Karatsuba, S.M. Voronin, The Riemann zeta-function, de Gruyter 1992. MR 1183467 (93h:11096)

[10] J. Steuding, On the value-distribution of the Hurwitz zeta-function at the nontrivial zeros of the Riemann zeta-function, Abhdlg. Math. Sem. Uni. Hamburg 71 (2001), 113-121. MR1872718 (2002i:11085)

[11] E.C. Titchmarsh, The theory of functions, Oxford University Press 1939.

[12] S. Wolfram, The Mathematica Book, Cambridge, England: Cambridge University Press, 1999, 4th ed. MR1721106 (2000h:68001)

Department of Mathematics and Informatics, Vilnius University, Naugarduko 24, 03225 Vilnius, LithuANiA

E-mail address: ramunas.garunkstis@maf.vu.lt

Institut für Mathematik, Würzburg University, Am Hubland, 97074 Würzburg, GerMANY

E-mail address: steuding@mathematik.uni-wuerzburg.de 